BULLETIN OF THE

AMERICAN MATHEMATICAL SOCIETY

Volume 81, Number 2, March 1975

\title{
CORRIGENDUM, VOLUME 79
}

P. Ponomarev, Class numbers of definite quaternary forms with nonsquare discriminant, pp. 594-598.

p. 595 , line 10 , replace " $n d>3$ " by " $n d \neq 1,3$ "

p. 596, line 17, after " $n d>3$," insert " $n d \neq D / 2$,"; replace " $d<D^{1 / 2}$ " by " $d$ is odd"

\section{CORRIGENDUM, VOLUME 80}

Norman Levinson, Zeros of derivative of Riemann's $\xi$-function, pp. 951-954.

The number 0.1414 appearing on the right of the last displayed inequality of [1] is too large. A trivial calculation shows it can be replaced by 0.1410 which yields a minor improvement in the final result. (Lowell Schoenfeld informs me that taking $R=1.08$, instead of 1.1 , leads to the improved lower bound of 0.7181792 for the proportion of zeros on $\xi^{\prime}(s)$ on $\sigma=1 / 2$. Presumably a semioptimal mollifier as in [2] would yield a better improvement.)

The second displayed formula of $[1$, p. 953] should be replaced by

$$
\int_{T}^{T+U} \log |\psi G(a+i t)| d t \leqslant U \log \left(\frac{1}{U} \int_{T}^{T+U}|\psi G(a+i t)| d t\right) .
$$

The integral on the right is then dominated by the sum of a main term and minor terms. The Schwarz inequality is then used on the main term to get a term of the form

$$
\left(\frac{1}{U} \int_{T}^{T+U}|\psi H(a+i t)|^{2} d t\right)^{1 / 2}
$$

\section{REFERENCES}

1. N. Levinson, Zeros of derivative of Riemann's $\xi$-function, Bull. Amer. Math. Soc. 80 (1974), $951-954$.

2. Deduction of semi-optimal mollifier for obtaining lower bound for $N_{0}(T)$ for Riemann's zeta-function, Proc. Nat. Acad. Sci. U.S.A. 72 (1975).

DEPARTMENT OF MATHEMATICS, MASSACHUSETTS INSTITUTE OF TECHNOLOGY, CAMBRIDGE, MASSACHUSETTS 02139 